PACS 64.70.Md, 61.30.Gd; UDC 532.783, 548-14

\title{
EXACT RELATIONS IN THE THEORY OF ANISOTROPIC LIQUIDS
}

\author{
T.G.SOKOLOVSKA, M.F.HOLOVKO \\ Institute for Condensed Matter Physics \\ of the National Academy of Sciences of Ukraine \\ 1 Svientsitskii St., 290011 Lviv-11, Ukraine
}

Received July 14, 1997; Revised version September 30, 1997

\begin{abstract}
Exact relations which connect the order parameters, $\boldsymbol{k}=0$ Fourier transforms of the direct correlation function harmonics and parameters of the single-particle distribution function for the uniaxial fluids are proposed. These relations are an algebraic represetation of the Lovett equation in anisotropic fluids. It is shown that in the anisotropic (orientationally ordered) phase the transverse correlations of spins in magnetic fluids and the correlations of the director transverse fluctuations in nematics become of long-range character.
\end{abstract}

Anisotropic fluids have no long-range positional order, but they do exhibit orientational order. As a consequence, the single-particle distribution function $\rho(1)$ does depend only on the molecular orientation $\omega=(\theta, \varphi)$ and $\rho(1)=\rho f\left(\omega_{1}\right)$, where $\rho$ is the density of molecules. These systems are polar liquids, magnetic fluids and the simplest of the liquid crystals - nematics. Anisotropic fluids without the presence of an external field are the systems with spontaneously broken symmetry, because the direction of the orientational ordering is not predetermined. The spontaneous ordering breaks the continuous symmetry (rotational invariance that is intrinsic in isotropic systems) and leads to orientational excitations, or Goldstone modes, which rotate the direction of ordering without any energy cost. This results in the peculiar physical properties of anisotropic fluids.

\section{Stability analysis and the Lovett equation in anisotropic fluids}

From the density functional theory the following stability condition of equilibrium systems is known [1]

$$
\iint \mathrm{d}(1) \mathrm{d}(2)\left[\frac{\delta(1,2)}{\rho(1)}-C(1,2)\right] \delta \rho(1) \delta \rho(2)>0,
$$

where $c(1,2)$ is the direct pair correlation function, 1 (or 2) being spatial and orientational coordinates of particle 1 (or 2). The equation (1.1) follows directly from the condition of the free energy minimum with respect to arbitrary variations in the particle distribution $\delta \rho(i)$ :

$$
\iint \frac{\delta^{2} F}{\delta \rho(1) \delta \rho(2)} \delta \rho(1) \delta \rho(2) \mathrm{d}(1) \mathrm{d}(2)>0
$$

But in the anisotropic fluids without the presence of external fields some variations (connected to rotations of the director: $\delta \rho(1) \sim \nabla_{\omega_{1}} \rho\left(\omega_{1}\right)$ ) keep

(C) T.G.Sokolovska, M.F.Holovko, 1997

ISSN 0452-9910. Condensed Matter Physics 1997 No 11 (109-115) 
the free energy of the system unchanged. It means that in (1.1) we should require equality for the corresponding variations. Therefore, one has to supplement the rigorous inequality (1.1) with the equality

$$
\frac{\nabla_{\omega_{1}} \rho(1)}{\rho(1)}-\int C(1,2) \nabla_{\omega_{2}} \rho(2) \mathrm{d}(2)=0 .
$$

The equation (1.3) is identical to the Lovett-Mou-Buff-Wertheim (Lovett) equation for [2] spatially uniform systems

$$
\nabla_{\omega_{1}} \ln \rho\left(\omega_{1}\right)+\nabla_{\omega_{1}} \frac{v\left(\omega_{1}\right)}{k_{\mathrm{B}} T}=\int C\left(\boldsymbol{r}, \omega_{1}, \omega_{2}\right) \nabla_{\omega_{2}} \rho\left(\omega_{2}\right) \mathrm{d} \omega_{2} \mathrm{~d} \boldsymbol{r}
$$

in the case of zero external field $v\left(\omega_{1}\right)$.

Thus, the Lovett equation is closely connected with the broken rotational invariance and correctly treats the symmetry of anisotropic fluids. We will show now that equation (1.4) also provides natural treatment for Goldstone modes in systems with spontaneously broken symmetry. In such systems the susceptibilities to external field $v$ rotations (transverse susceptibilities) are infinite in the zero-field limit. In anisotropic fluids the orientation of the molecules breaks the continuous rotational symmetry (but not the translational invariance) and results in a spontaneous partial order. Therefore [3], the transverse susceptibility in anisotropic fluid is infinite in the limit of a zero wave vector. As the total pair correlation function $h\left(\boldsymbol{k}, \omega, \omega^{\prime}\right)$ is directly related to the susceptibility, then $h\left(\boldsymbol{k}, \omega, \omega^{\prime}\right)$ also becomes infinite as $k \rightarrow 0$. In [3] it was shown from the Ornstein-Zernike (OZ) equation that $h\left(\boldsymbol{k} \rightarrow 0, \omega, \omega^{\prime}\right) \rightarrow \infty$ corresponds to the existence of a unit eigenvalue of the integral operator $\tilde{C}\left(\boldsymbol{k}=0, \omega_{1}, \omega_{2}\right)=\rho^{1 / 2}\left(\omega_{1}\right) \int C\left(\boldsymbol{r}, \omega_{1}, \omega_{2}\right) \mathrm{d} \boldsymbol{r} \rho^{1 / 2}\left(\omega_{2}\right)$. One can see that the Lovett equation (1.4) for $v\left(\omega_{1}\right)=$ const is an eigenequation for the operator $\tilde{C}\left(\boldsymbol{k}=0, \omega_{1}, \omega_{2}\right)$

$$
\int \mathrm{d} \omega_{1} \tilde{C}\left(\boldsymbol{k}=0, \omega, \omega_{1}\right) \psi_{i}\left(\boldsymbol{k}=0, \omega_{1}\right)=\lambda_{i}(\boldsymbol{k}=0) \psi_{i}(\boldsymbol{k}=0, \omega)
$$

with eigenvector $\psi_{i}(\boldsymbol{k}=0, \omega)=\rho^{-1 / 2}(\omega) \nabla_{\omega} \rho(\omega)$ and unit eigenvalue $\lambda_{i}(\boldsymbol{k}=$ $0)=1$. Therefore, the direct correlation function and the single-particle distribution function complying with the Lovett and $\mathrm{OZ}$ equations treat correctly Goldstone modes in the system. But there are great difficulties in the obtaining of a self-consistent solution to this system of equations. The most difficult confusion is connected with the treatment of the integrodifferential Lovett equation. In the next section we present the algebraic representation of the Lovett equation for an uniaxial fluid in the form of exact relations. It will be shown that this representation correctly treats Goldstone modes.

\section{Exact algebraic representation of the Lovett equation in uniaxial fluids}

In uniaxial fluids the orientational distribution function $f(\omega)$ is axially symmetric around a preferred direction $\boldsymbol{n}$ and depends only on the angle $\theta$ between the molecular orientation $\omega$ and $\boldsymbol{n}$. It allows us to write $f(\omega)$ in the 
form

$$
f(\omega)=\frac{1}{Z} \exp \left\{\sum_{l>0} A_{l} Y_{l 0}(\omega)\right\},
$$

where the constant $Z$ can be found from the normalization condition

$$
\int f(\omega) \mathrm{d} \omega=1
$$

Spherical harmonics $Y_{l m}(\omega)$ satisfy the standard Condon and Shortley phase convention [4].

The nematic ordering is defined by the parameters

$$
S_{l}=\left\langle P_{l}(\cos \theta)\right\rangle=\int \mathrm{d} \omega f(\omega) P_{l}(\cos \theta),
$$

where $P_{l}(\cos \theta)$ are the Legendre polynomials.

In this note we shall discuss the exact relations between $S_{l}, A_{l}$ and

$$
C_{m n}^{\mu \mu}=\frac{1}{\sqrt{4 \pi}} \int \mathrm{d} \boldsymbol{r} C_{m n 0}^{\mu \mu 0}(r)
$$

where $C_{m n l}^{\mu \nu \lambda}(r)$ are the coefficients of the orientational expansion of the direct correlation function $C\left(\boldsymbol{r}, \omega_{1}, \omega_{2}\right)$.

In the space-fixed coordinate system with $z$-axis parallel to $\boldsymbol{n}$ the pair direct correlation function of linear molecules can be written in the form

$$
C\left(\boldsymbol{r}, \omega_{1}, \omega_{2}\right)=\sum_{m, n, l, \mu, \nu, \lambda} C_{m n l}^{\mu \nu \lambda}(r) Y_{m \mu}\left(\omega_{1}\right) Y_{n \nu}^{*}\left(\omega_{2}\right) Y_{l \lambda}\left(\omega_{\boldsymbol{r}}\right),
$$

$r$ is a separation-vector of molecules mass centres, $\omega_{\boldsymbol{r}}$ being its orientation. It should be noted, that for axially symmetric system $\mu+\lambda=\nu$.

For our purposes we use the Lovett-Mou-Buff-Wertheim equation which for anisotropic fluids can be written in the form [5]

$$
\nabla_{\omega_{1}} \ln \rho\left(\omega_{1}\right)=\int C\left(\omega_{1}, \omega_{2}\right) \nabla_{\omega_{2}} \rho\left(\omega_{2}\right) \mathrm{d} \omega_{2}
$$

where $C\left(\omega_{1}, \omega_{2}\right)=\int C\left(\boldsymbol{r}, \omega_{1}, \omega_{2}\right) \mathrm{d} \boldsymbol{r}, \nabla_{\omega}$ is the angular gradient operator for a linear molecule. The space-fixed $X, Y$ and $Z$ components are given by $\nabla_{\omega}=i \boldsymbol{l}$, where $\boldsymbol{l}$ is the angular momentum operator. Using the relations $[4]$

$$
\begin{gathered}
\left(\nabla_{\omega}\right)_{y}=\frac{\hat{l}_{+}-\hat{l}_{-}}{2}, \\
\hat{l}_{ \pm} Y_{m \mu}(\omega)=[m(m+1)-\mu(\mu+1)]^{1 / 2} Y_{m \mu \pm 1}(\omega)
\end{gathered}
$$

and expansions $(2.1),(2.4)$ the $y$-component of $(2.5)$ is obtained in the form:

$$
\begin{aligned}
& \sum_{M>0} \sqrt{M(M+1)} A_{M}\left[Y_{M 1}\left(\omega_{1}\right)-Y_{M-1}\left(\omega_{1}\right)\right]= \\
& =\sum_{M^{\prime}>0} \sum_{m, n, \mu} \int C_{m n}^{\mu \mu} Y_{m \mu}\left(\omega_{1}\right) Y_{n \mu}^{*}\left(\omega_{2}\right) \sqrt{M^{\prime}\left(M^{\prime}+1\right)} A_{M^{\prime}} \times \\
& \times\left[Y_{M^{\prime} 1}\left(\omega_{2}\right)-Y_{M^{\prime}-1}\left(\omega_{2}\right)\right] \rho\left(\omega_{2}\right) \mathrm{d} \omega_{2} .
\end{aligned}
$$


Taking into account that only independent of the azimuthal angle $\varphi$ quantities yield nonzero average values and using the orthogonality of $Y_{l m} \mathrm{~s}$, one gets the following matrix equation

$$
\boldsymbol{L}=\hat{C} \hat{Y} \boldsymbol{L},
$$

where $\boldsymbol{L}$ is a column consisting of $L_{M}=\sqrt{M(M+1)} A_{M}, \hat{C}$ and $\hat{Y}$ are of order $(N \times N)$ with matrix elements $C_{m n}^{11}$ and $Y_{m n}^{11}$, where

$$
Y_{m n}^{11}=\rho \int \mathrm{d} \omega f(\omega) Y_{m 1}(\omega) Y_{n 1}^{*}(\omega)
$$

can be expressed via $S_{l}, N$ is a number of values of index $m$ (or $n$ ) such that $C_{m n}^{11} \neq 0$.

Since the angular momentum operator $\boldsymbol{l}$ is hermitian we can write eq. (2.5) in the equivalent form:

$$
\nabla_{\omega_{1}} \ln \rho\left(\omega_{1}\right)=-\int\left[\nabla_{\omega_{2}} C_{2}\left(\omega_{1}, \omega_{2}\right)\right] \rho\left(\omega_{2}\right) \mathrm{d} \omega_{2} .
$$

In the similar manner from (2.11) we can obtain a matrix equation for the coefficients of the function $f(\omega)$

$$
\boldsymbol{L}=\hat{C} \boldsymbol{P},
$$

where $\boldsymbol{P}$ contains order parameters of the system:

$$
P_{l}=\frac{\rho \sqrt{l(l+1)} \sqrt{2 l+1}}{\sqrt{4 \pi}} S_{l} .
$$

Thus, the symmetries of the angular gradient operator and the nematic system yield two matrix relations (2.9) and (2.12) which connect the system order parameters, zero Fourier transforms of the direct correlation function harmonics and coefficients $A_{l}$ of the single-particle distribution functions of the system.

Joint use of (2.9) and (2.12) allows us to express $f(\omega)$ via the order parameters only:

$$
\boldsymbol{L}=\hat{Y}^{-1} \boldsymbol{P}
$$

The obtained relations can be very useful for the calculation of the correlation functions of the anisotropic phase and serve to verify the fitness of chosen models and approximations. For the illustration we suppose that the expansion (2.4) may contain at $Y_{00}\left(\omega_{\boldsymbol{R}}\right)$ only $Y_{m \mu}\left(\omega_{1}\right), Y_{n \mu}^{*}\left(\omega_{2}\right)$ with $m, n=0,2$. Then the eq. (2.9) and (2.12) yield

$$
\begin{array}{ll}
4 \pi\left(\rho C_{22}^{11}\right)^{-1} & =\frac{4 \pi Y_{22}^{11}}{\rho}=1+\frac{5}{7} S_{2}-\frac{12}{7} S_{4}, \\
\left(\rho C_{22}^{11}\right)^{-1} & =\sqrt{\frac{5}{4 \pi}} \frac{S_{2}}{A_{2}}
\end{array}
$$

For the isotropic $S_{2}=S_{4}=0, Y_{22}^{11}=\frac{\rho}{4 \pi}$, and the first of eq. (2.15) brings out the instability point of the isotropic phase (that is identical to the result of the density functional theory $[6])$ :

$$
1=(4 \pi)^{-\frac{3}{2}} \rho \int C_{220}^{110(i s o)}(r) \mathrm{d} \boldsymbol{r},
$$


$C_{220}^{110(i s o)}(r)$ is the corresponding harmonic of the isotropic direct correlation function. As the single-particle distribution function in this case has the Mayer-Saupe form, we equate $\frac{4 \pi Y_{22}^{11}}{\rho}$ with the first non-trivial value of the self-consistency equation $\frac{4 \pi Y_{22}^{11}}{\rho}=1.1142$ with $S_{2}=0.3236$ [7] and obtain the bifurcation point of the nematic solution for our model:

$$
1.1142^{-1}=(4 \pi)^{-\frac{3}{2}} \rho \int C_{220}^{110(n e m)}(r) \mathrm{d} \boldsymbol{r},
$$

where $C_{220}^{110(\text { nem })}(r)$ is the corresponding harmonic of the direct correlation function for the nematic phase. It is worth to note that equations (2.15) determine an independent of $A_{2}$ relation between $S_{2}$ and $S_{4}$ since in this case $A_{2}$ is a single-valued function of $S_{2}$ (see (2.2)). The same situation is observed in the computer simulation for the system of thin hard platelets [8].

The task becomes more complicated if $C_{m n}^{11} \neq 0$ for several values of $m$ and $n$, for instance, 1 and 2. Now $\hat{C}$ is a nonsingular matrix $2 \times 2$. Using relation $(2.14)$ we get

$$
\left(\begin{array}{c}
\sqrt{2} A_{1} \\
\sqrt{6} A_{2}
\end{array}\right)=\sqrt{4 \pi}\left(\begin{array}{cc}
1-S_{2} & \frac{3}{\sqrt{5}}\left(S_{1}-S_{3}\right) \\
\frac{3}{\sqrt{5}}\left(S_{1}-S_{3}\right) & 1+\frac{5}{7} S_{2}-\frac{12}{7} S_{4}
\end{array}\right)^{-1}\left(\begin{array}{c}
\sqrt{6} S_{1} \\
\sqrt{30} S_{2}
\end{array}\right) .
$$

Equation (2.12) takes the form

$$
\left(\begin{array}{l}
\sqrt{2} A_{1} \\
\sqrt{6} A_{2}
\end{array}\right)=\frac{\rho}{\sqrt{4 \pi}}\left(\begin{array}{ll}
C_{11}^{11} & C_{12}^{11} \\
C_{21}^{11} & C_{22}^{11}
\end{array}\right)\left(\begin{array}{c}
\sqrt{6} S_{1} \\
\sqrt{30} S_{2}
\end{array}\right) .
$$

Equations (2.18) and (2.19) yield

$$
\begin{aligned}
& 4 \pi\left(\begin{array}{cc}
1-S_{2} & \frac{3}{\sqrt{5}}\left(S_{1}-S_{3}\right) \\
\frac{3}{\sqrt{5}}\left(S_{1}-S_{3}\right) & 1+\frac{5}{7} S_{2}-\frac{12}{7} S_{4}
\end{array}\right)^{-1}\left(\begin{array}{c}
\sqrt{6} S_{1} \\
\sqrt{30} S_{2}
\end{array}\right)= \\
& =\rho\left(\begin{array}{ll}
C_{11}^{11} & C_{12}^{11} \\
C_{21}^{11} & C_{22}^{11}
\end{array}\right)\left(\begin{array}{c}
\sqrt{6} S_{1} \\
\sqrt{30} S_{2}
\end{array}\right) .
\end{aligned}
$$

One can see that equation (2.20) determines $\hat{C}$ via the order parameters as soon as the cross terms $C_{12}^{11}$ and $C_{21}^{11}$ are negligibly small. In a general case we have two equations for three unknown values $C_{11}^{11}, C_{12}^{11}=C_{21}^{11 *}$ and $C_{22}^{11}$.

In order to examine the exact relations we consider the $\mathrm{OZ}$ equation for anisotropic fluids:

$$
h(1,2)=C(1,2)+\int \rho(3) C(1,3) h(3,2) \mathrm{d}(3),
$$

where $C(1,2)$ and $h(1,2)$ are the direct correlation function and the pair correlation function respectively; $d 3=d \boldsymbol{R}_{3} d \omega_{3}$, where $\boldsymbol{R}$ is mass center coordinates of the particle, $\omega=(\theta, \varphi)$ means the orientation of the molecule. As $\rho(3)$ does not depend on $\boldsymbol{R}_{3}$ then the Fourier-component $(\boldsymbol{k}=0)$ of $(2.21)$ can be rewritten in the form of the matrix equations:

$$
\hat{H}^{(\mu)}=\hat{C}^{(\mu)}+\hat{C}^{(\mu)} \hat{Y}^{(\mu)} \hat{H}^{(\mu)},
$$


where

$$
\begin{gathered}
H_{m n}^{(\mu)}=\int \mathrm{d} \boldsymbol{R} h_{m n 0}^{\mu \mu 0}(R), \\
C_{m n}^{(\mu)}=\int \mathrm{d} \boldsymbol{R} C_{m n 0}^{\mu \mu 0}(R), \\
Y_{m n}^{(\mu)}=\int \mathrm{d} \omega f(\omega) Y_{m \mu}(\omega) Y_{n \mu}^{*}(\omega) .
\end{gathered}
$$

One can verify (2.22) taking into account that the uniaxial fluid is invariant with respect to rotations around $z$ axis.

Equation (2.22) can be presented in the form:

$$
\sqrt{Y^{(\mu)}} \hat{H}^{(\mu)} \sqrt{Y^{(\mu)}}=\left\{1-\sqrt{Y^{(\mu)}} \hat{C}^{(\mu)} \sqrt{Y^{(\mu)}}\right\}^{-1} \sqrt{Y^{(\mu)}} \hat{C}^{(\mu)} \sqrt{Y^{(\mu)}} .
$$

The matrix $\hat{H}^{(\mu)}$ is singular if $\operatorname{det}\left(1-\sqrt{Y^{(\mu)}} \hat{C}^{(\mu)} \sqrt{Y^{(\mu)}}\right)=0$. The latter condition is satisfied for $\mu=1$ in uniaxial fluids due to the obtained relation (2.9). Thus, Goldstone modes are connected with the corresponding harmonics of the pair correlation function $\left(h_{m n 0}^{110}(\boldsymbol{k}=0)\right)$. Since these harmonics are coupled with the transverse correlations of spins in magnetic fluids and the correlations of the director transverse fluctuations in nematics, the peculiarities $h_{m n 0}^{110}(\boldsymbol{k}=0) \rightarrow \infty$ are responsible for a long-range character of the above-mentioned correlations in the ordered phases.

\section{Acknowledgement}

This work was partially supported by the State Committee for Science and Technology of Ukraine (project No 2.3/371)

\section{References}

[1] Lipszyc K., Klockowski A. The isotropic-nematic phase transition as the bifurcation of solutions of the nonlinear integral equation. // Acta Physica Polonica, 1983, vol. A63, No 6, p. 805-822.

[2] Wertheim M.S. Correlations in the liquid-vapor interface. // J. Chem. Phys., 1976, vol. 65, No 6, p. 2377-2381.

[3] Zhong H., Petschek R. Percus-Yevick approximation for fluids with spontaneous partial order: Results for a separable model. // Phys. Rev. E, 1996, vol. 53, No 5, p. 4944-4954..

[4] Gray C.G., Gubbins K.E. Theory of molecular fluids, v. 1: Fundamentals (Clarendon press, Oxford, 1984).

[5] Rowlinson J.S., Widom B. Molecular theory of capillarity (Clarendon press, Oxford, 1982).

[6] Klochkowski A., Stecki J. On the mean spherical model for liquid crystals. // Mol. Phys., 1982, vol. 46, No 1, p. 13-19.

[7] Sokolovska T.G., Holovko M.F., Sokolovskii R.O. Phase diagrams for a model of nematic with long-range anisotropic potential: The mean spherical approximation. Ukr. Phys. J., in print.

[8] Frenkel D. Statistical mechanics of liquid crystals. In: Liquids, Freezing and Glass Transition, eds. J.P.Hansen, D.Levesque and J. Zinn-Justin (Elsevier, Amsterdam, 1991), ch. 9, p. 690-762. 


\title{
ТОЧНІ СПІВВІДНОШЕННЯ \\ В ТЕОРІЇ АНІЗОТРОПНИХ РІДИН
}

\author{
Т.Г.Соколовська, М.Ф.Головко
}

Для одноосних плинів запропоновано точні співвідношення, що пов'язують параметри порядку, фур'є-образи гармонік прямої кореляційної функції при $\boldsymbol{k}=0$ і параметри одночастинкової функції розподілу. Ці співвідношення є алгебраїчним представленням рівняння Ловетта для анізотропних плинів. Показано, що в анізотропній (орієнтаційно впорядкованій) фазі поперечні кореляції спінів у магнітних рідинах і кореляції поперечних флуктуацій директора у нематиках набувають далекосяжного характеру. 